with the help of Poisson's elastic constant and is in perfect agreement with the experimental data available. The theoretical derivation uses a kind of thermodynamical equilibrium which differs from the usual methods; this is due to the fact that a solid under stress is not a genuine statistical equilibrium. This question needs further investigation though one can scarcely doubt the correctness of the resultant formula. The view due to Smekal that breaking strength belongs to the structure-sensitive (strukturempfindlich) properties of solids must be abandoned, since it can be derived by thermodynamical reasoning without even mentioning atomic structure. MAX BORN.

Department of Applied Mathematics, University of Edinburgh.

\section{An Electron Diffraction Study of the} Surfaces of Alkali and Alkaline Earth Metals Exposed to Air

THE most corrodible metals are those of the alkali and the alkaline earth groups. The products forming on a freshly cut surface of these metals on exposure to air were studied.

A fresh clean surface of lithium was allowed to stand in air for about five minutes. The diffraction pattern obtained from such a surface clearly showed the formation of lithium oxide $\left(\mathrm{Li}_{2} \mathrm{O}\right)$ mixed with a small amount of lithium hydroxide.

The pattern obtained from the surface of sodium similarly treated showed the formation of sodium bicarbonate; a micro-chemical analysis also verified the presence of the $\mathrm{CO}_{3}^{--}$radical.

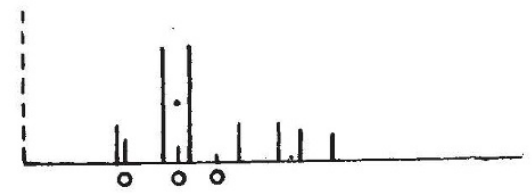

DIFFRACTION PATTERN FROM LITHIUM SPECIMEN. O, LIOH ; OTHER LINES, $\mathrm{LI}_{2} \mathrm{O}$.

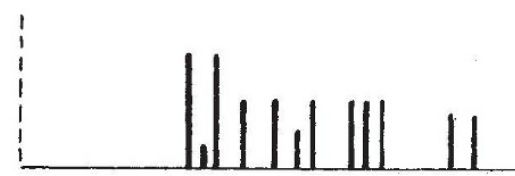

PATTERN FROM SODIUM SPECTMEN, SHOWING MAINLY THE EXISTENCE OF $\mathrm{NAHCO}_{3}$.

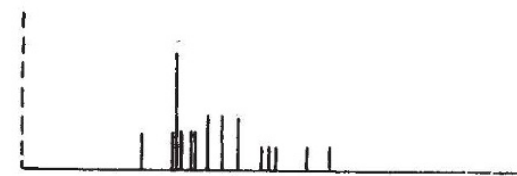

PATtern From POTASSIUM SPECIMEN, SHOWING MAINLY THE EXISTENCE OF $\mathrm{KHCO}_{3}$.

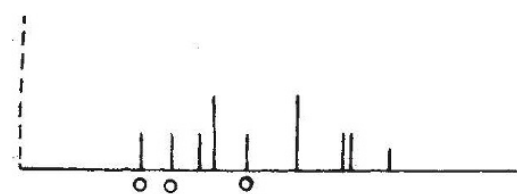

THE PATTERN FROM CAICIUM SPECIMEN. o, CA ; OTHER LINES, CaO.
The formation of potassium bicarbonate on the surface of potassium in similar circumstances also occurs, but in this case the diffraction rings are sharper than those of sodium bicarbonate on sodium; this suggests that the reaction producing potassium bicarbonate is faster than that producing sodium bicarbonate.

Similarly, a moderate quantity of calcium oxide (CaO) was found to be formed on the surface of calcium exposed to air for about five minutes. On comparing this pattern with that obtained in the magnesium experiment under similar conditions ${ }^{1}$, it is apparent that the oxide film on ealcium is much thicker than that on magnesium.

These results reveal the fact that the carbon dioxide in air can act on potassium or sodium within the limited period of about five minutes, but not on lithium or calcium; while oxygen and the moisture in air act on all metals here investigated.

Full details of these experiments will be published in the Scientific Papers of this Institute. The work has been conducted under the direction of Dr. Ichiro Titaka.

Institute of Physical and

Shigeto Yamaguchr.

Chemical Research, Tokyo.

${ }^{1}$ litaka, I., and Yamaguchi, S., NATURE, 144, 1090 (1 939) ; Yamaguchi' S., Sci. Pap. Inst. Phys. Chem. Research, 36, 463 (1939).

\section{Separation of Uranium Isotopes}

THe separation of the uranium isotopes is of interest because only one of the isotopes, ${ }^{235} \mathrm{U}$, is believed to undergo fission when bombarded by slow neutrons. The best way to check this theory would be to change the relative particle density of ${ }^{235} \mathrm{U}$ and to see if that has any influence on the number of fissions caused by a certain number of neutrons. If the theory proves correct, one might be able to cause a nuclear chain reaction by concentrating ${ }^{235} \mathrm{U}$ and removing the other isotopes, which only catch neutrons without producing new ones.

To begin with, the most suitable method for concentrating ${ }^{236} \mathrm{U}$ seems to be the thermal-diffusion method of Clusius and Dickel ${ }^{1}$ or Brewer andBramley ${ }^{2}$, this method being a universal one, applicable to all elements. Of course, other methods, specifically suitable for uranium, may be found later on. The thermal diffusion method can be used both for liquids and for gases, but it works extremely slowly when applied to liquids. It is much better to use gases. The only volatile compound of uranium is uranium fluoride $\left(\mathrm{UF}_{6}\right)$. The dimensions of a thermal-diffusion tube system, using this compound, can be calculated from the formulæ of Furry, Jones and Onsager ${ }^{3}$. We shall use the same notation as these authors.

The molecular weight of uranium fluoride being 352 , the factor $\alpha$ is about $1.5 \times 10^{-3}$. The temperature of the colder outer tube must be about $T_{1}=333^{\circ} \mathrm{K}$. $=60^{\circ} \mathrm{C}$., if the apparatus is run at ordinary pressure, as uranium fluoride is a solid below $59^{\circ} \mathrm{C}$. at a pressure of $760 \mathrm{~mm}$. mercury. The temperature $T_{2}$ of the hot tube can be about $2 T_{1}=$ $666^{\circ} \mathrm{K} .=393^{\circ} \mathrm{C}$, as experiments have been made with uranium fluoride at $440^{\circ} \mathrm{C}$. without decomposition of the gas. Too high a temperature would 